\title{
The Canadian Medical Association Journal (CMA) would like to thank the following peer reviewers who contributed their time and expertise to the Journal in 2006. The contribution of these individuals has been vital to enhancing the quality of articles published in CMAJ and advancing the discourse of medicine.
}

Aaron, S.D.

Abdulla, Aly

Aberman, Arnie

Abi-Jaoude, Elia

Abrams, J.

Adamek, Milan

Adang, E.

Addison, Clifton C.

Adetunji, Babatunde

Afilalo, Jonathan

Agarwal, Gina

Aghaei, Shahin

Ahmed, S. Nizam

Alberti, Luiz Ronaldo

Alexander, G. Caleb

Alfieri, Carolina

Alibhai, Shabbir M.H.

Alkhenizan, Abdullah

Allan, G. Michael

Allen, Kelli D.

Alliot, Carol

Allotey, Pascale

Almoosa, Khalid

Alonzo, Todd

Alouf, Benjamin

Anastassiades, Tassos

Andermann, Frederick

Andersen, H.R.

Andersen, Ole

Anderson, David R.

Anderson, John E.

Anderson, Robert Paul

Anderson, Sarah

Angel, Jonathan

Angulo, Paul

Anton, Hubert

Aoki, Fred Yoichi

Arbogast, Kristy B.

Arendt, Mikkel

Argent, Nicholas Boyd

Armstrong, Irene

Armstrong, Paul W.

Arseneault, Louise

Asaro, Phillip V.

Asztalos, Elizabeth

Atlas, Michel C.

Auer, J.

Aujesky, Drahomir Auray-Blais, Christiane

A Avinashi, Vishal

Aylin, Paul

Azzazy, Hassan M.E.

Back, Anthony

Bains, Namrata

Baker, Steven Kent

Balkrishnan, Rajesh
Ballantyne, Jane Carol

Balneaves, Lynda G.

Banatvala, J.E.

Banerji, Anna

Baran, Dana

Barnard, C.B.

Barnett, Henry Joseph

Barnett, Steven

Barr, J.K.

Barratt, Jonathan

Barrett, Brendan J.

Bartalena, L.

Bartoli, Ettore

Baruch, Lawrence

Baskett, Roger J.F.

Bassotti, Gabrio

Bates, Joanna Margaret

Baum, Michael

Baxter, Susan

Baylis, Francoise

Bazarian, Jeff

Beach, Jeremy

Beaulieu, P.

Beck, Cynthia Anita

Beck, David E.

Becker, Harold

Beghi, Ettore

Behn, Agnieszka Dorota

Behrens, Ron

Beletsky, Vadim

Bell, Alan David

Bell, D.S.H.

Bell, Mary

Bellan, Lorne

Bellentani, Stefano

Bemos, Dale J.

Benenson, Shmuel

Berger, Philip Benjamin

Bern, Caryn

Bernstein, Alan

Bernstein, S.L.

Bertone Johnson, E.R.

Beskow, Laura M.

Betran, A.P.

Beynon, C.M.

Bian, J.

Biderman, Aya

Bidus, Michael

Bielory, Leonard

Biffl, Walter L.

Bilukha, Oleg O.

Birnie, David Hugh

Birtwhistle, Richard Vernon

Bjerre, Lise $\mathrm{M}$.

Bjordal, Jan M.

Blackmer, Jeff
Bland, Roger

Bleich, Avi

Bloch, Ralph F.

Blum, Arnon

Bluthenthal, Ricky

Bobrik, Alexey

Bock, N.N.

Boersma, Eric

Bogenschutz, M.P.

Bongiavanni, Marco

Bork, Konrad

Borrie, Michael J.

Bortolussi, Robert

Borwein, Sarah T

Bosch, Xavier

Boufous, Soufiane

Bouhassira, Didier

Boustcha, E.M.

Bovet, Pascal

Bowen, Tom

Bowie, William R.

Boyd, C.

Boyle, Robert

Bradley, E.H.

Braidy, Joseph

Braitstein, Paula

Bramstedt, Katrina

Brass, E.P.

Brassard, Paul

Braun, J.

Brennan, M.

Bresser, Paula J.C.

Bressler, Brian

Brice, Julie

Bridges, F. Stephen

Brinton, Louise A.

Brock, Gerald B.

Bronfort, Gert

Brophy, James MacKay

Brophy, Jason Clarence

Brown, Celia

Brown, T. Desmond

Brownstein, John

Brunham, Robert C.

Buchanan, Michael R.

Buckeridge, D.L.

Buetow, Stephen

Bugianesi, Elisabetta

Bujold, Emmanuel

Bunin, G.R.

Burd, Larry

Burge, Fred

Burgess, John H.

Burgess, Lesley Jean

Burke, R.J.

Burkle, F.M.
Burns, L.

Busse, Jason Walter

Butler, Javed

Byszewski, Anna

Cademartiri, Filippo

Cadesky, Eric

Calabrese, Leonard

Callaghan, R. C.

Calzavara, Liviana M.

Cameron, D. William

Cantarovich, Marcelo

Cantor, Warren Joseph

Capek, Radan

Caplan, Gideon A.

Cappato, Riccardo

Carceller Garcia, Ana Maria

Carette, Simon

Caro, Jaime

Caron, Nadine Rena

Carrie, Anita

Carter, Anne Ora

Casais, Patricia

Cass, Alan

Castle, David Jonathan

Cawthorpe, David

Cecatti, Jose Guilherme

Chalmers, Andrew

Chambers, L.W.W.

Chan, An-Wen

Chapman, Alex

Charland, Louis C.

Charney, Dara

Chaturvedi, Santosh K.

Chee, Victor

Chen, Alice W.

Chen, Tzeng-Ji

Cherniak, Donna

Chervenak, Frank

Chevalier, Serge

Chiappelli, Francesco

Chin-Yee, Ian Harold

Chipman, Mary L.

Cho, Juhee

Choi, Bernard C.K.

Chow, Kai Ming

Christensen, Helle Collatz

Chudley, Albert Edward

Chui, Linda

Chung, Betty P.M.

Chung, F.M.

Ciatto, Stefano

Clarfield, A. Mark

Clark, C. Graham

Clarke, Gwen

Clarke, Linda E.

Cobo, Erik
Cole, Edward

Cole, Madeleine

Cole, Martin G.

Coleman, Christopher

Colgrove, J.

Collinson, Paul

Conly, John Maynard

Cooper, Andrew B.

Cooper, Austin Rick

Cooper, Duncan

Cooper, Richard Michael

Corden, Timothy E.

Correa-de-Araujo, Rosaly

Corriveau, André

Corti, Marcelo

Costello-Boerrigter, Lisa C.

Cowie, Robert Lawrence

Cram, Peter

Cramer, J. Steven

Cranney, Ann

Creery, David

Crelinsten, Gordon L.

Crespo, Carlos

Croll, J.K.

Crosbie, D.J.

Cruess, Alan F.

Cuervo, Luis Gabriel

Cullen, Nora

Cummings, Beth-Ann M.

Cummings, Michael

Curnow, W.J.

Curns, Aaron T.

Cuttini, Marina

Dagenais, Simon

Dahle, Ulf R.

Dahlgren, Leanne

Dalby, Thomas

Dale, Krista

Daly, Donald S.

D'Amico, G.

Danzi, Sara

D'Arcy, Carl

Das, Undurti N.

da Silva, Benedito Borges

Dattani, Mehul Tulsidas

Davidson, M.B.

Davies, Elizabeth Anne

Davis, Alvin E.

Davis, Kepler A.

Davis, Peter

Day, Anna

Deber, Raisa B.

Deck, Wilber

Declercq, Eugene

Decosas, Josef

de Costa, C.M. 
Decullier, Evelyne

De Giorgi, Vincenzo

Dehghani, Payam

Deitrick, L.

Dekkers, Caroline

Delaney, J.A.C.

de Lima, $M$.

Delisle, Hélène

Dell, Sharon

DeMets, D.L.

Demianczuk, N.N.

Demmy, Todd

Denisco, Richard

Denomme, Greg

De Palma, Luigi

Depew, William T.

Derrick, J.L.

Desapriya, Ediriweera

Deshpande, Vikram

Des Jarlais, Don Conway

de Tayrac, Renaud

Detsky, Allan S.

Devault, K.R.

Dial, Mary Sandra

Diamanti-Kandarakis, E.

Diamond, George

Dickens, Bernard

Dickstein, Kenneth

Diel, Roland

Diener, $\mathrm{H}$.

Dilazzaro, Vincenzo

Dincer, I.

Dionne, Clermont E.

Discigil, Guzel

Djernes, J.K.

Djulbegovic, Benjamin

Dodd, J.

Doig, Christopher James

Doja, Asif

Dokainish, Hisham

Donohoe, Martin Thomas

Dooley, J.

Doty, Timothy Philip

Dow, Jamie

Dowd, M.D.

Downing, Mark Andrew

Dubé, Serge

Duff, Patrick

Duncan, Robin Elaine

Dunn, Sheila Frances Mary

Dyck, Peter

Ebbesson, Sven

Eckstein, $M$.

Ehrlich, P.F.

Einstein, Gillian

Elbourne, Diana

Ellard, David R.

Ellenbogen, Ken Alan

Ells, Carolyn

El Menyar, Ayman Ahmed

Engdahl, Johan

Enkin, Murray W.

Ernst, Pierre

Escobar-Morreale, H.F.

Essebag, Vidal

Evans, Gerald Anthony

Evenson, D.P.
Fabbri, A.

Fabbri, Paolo

Falguera, Miquel

Fanning, E. Anne

Fedele, Stefano

Fekkes, M.

Feldman, Debbie

Ehrmann

Feldman, Marc D.

Fellinger, Johannes

Fergusson, Dean A.

Ferris, Lorraine $\mathrm{E}$.

Filion, Lionel G.

Finberg, L.

Fine, Robert Lee

Finer, L.

Fingerote, Robert Joel

Finkelstein, Jonathan A.

Finkelstein, Yaron

Fisher, Alexander A.

Fisher, Paul Douglas

Fisher, William A.

Fisman, David Norman

Fitchett, David Hugh

Fitzcharles, Mary-Ann

Flanagin, Annette

Fleetham, John

Fleury, Manon

Flickinger, B.D.

Fodor, George

Forbes, Geoffrey M.

Forbes, Thomas Lawrence

Ford-Jones, Elizabeth Lee

Forssell, Heli

Fortenberry, J.D.

Foster, Beth J.

Foster, D.G.

Fox, Gary Norman

Franco, Eduardo L.

Frank, Blye

Franks, Peter

Fraser, Christophe

Fraser, J.D.

Freedman, John

Freiman, Anatoli

Frohlich, Jiri

Fugh-Berman, Adriane

Fulda, Gerard J.

Furlan, Andrea D.

Furtado, G.H.

Gabrielli, A.F.

Gafni, Amiram

Gagnier, Joel

Gajic, O.

Galanis, Eleni

Galley, Helen F.

Galloway, Mike

Gallus, Silvano

Garcia Rodriguez, L.A.

Gardam, Michael Andrew

Gareri, Joey Nicky

Garg, Amit

Gawaz, Meinrad

Gaziano, J.M.

Gemmill, Ian M.

Gemzell-Danielsson, K.

Genuis, Stephen J.
Genuth, S.

Gerritsen, A.

Geuze, S.G.

Ghali, Peter

Ghali, William Amin

Ghanizadeh, Ahamd

Ghoniem, Gamal M.

Giannetti, Nadia

Giannini, E.G.

Gibney, Noel

Gilbert, Nicolas L.

Gilfix, Brian M.

Gill, Sabrina

Gillespie, Ian

Gilmour, Joan

Girardi, E.

Gladstein, J.

Glasser, John W.

Gluud, Lise Lotte

Godwin, Marshall

Goh, Y. Ingrid

Gold, Ronald

Goldberg, Jay

Goldberg, Peter

Goldbloom, David Samuel

Goldman, Ran D.

Goldsand, George

Goldstein, Michael

Gonzalez, Francisco

Goodacre, Steve

Gorfinkel, Iris Shasha

Gorincour, Guillaume

Gottesman, I.I.

Gottesman, Ronald

Goyer, Robert

Graham, M.M.

Graham, Stephen D.

Grandmaison, Anne

Grantmyre, John

Granton, John T.

Granwehr, Bruno Palma

Gray, Jean D.

Green, Martin

Greenberg, David

Greenberg, Mark L.

Greenwald, Beverly

Greer, Julia Butler

Grepmair, L.J.

Greyson, Bruce

Griffiths, Anthony Neil

Gross, Douglas P.

Grosse, Scott

Grover, Steven

Grunau, Gilat Linn

Grunfeld, Eva

Guest, Cameron

Guglin, Maya

Gulliford, $M$.

Gunduz-Bruce, Handan

Guo, Ruoling

Gupta, Milan

Gupta, Prem Parkash

Gwadry-Sridhar, Femida

Hachulla, Eric

Hackam, Daniel G.

Hagel, Brent E.

Haider, Shariq
Halasi, Susan

Haldane, David

Hall, Judith G.

Hall, Philippa P. Grace

Hallander, Hans

Halpern, Scott D.

Hamalainen, $M$.

Hameed, Morad

Hamilton, Jill

Hammond, David

Hammond, Greg

Han, Xiang Y.

Hansen, Lisa

Hansson Mild, K.

Hardell, Lennart

Harris, M.F.

Harrison, Christine

Harrison, Rosamund

Harrold, Leslie

Hart, Trevor Adam

Harth, Manfred M.

Hasselback, Paul D.

Hatipoglu, Osman

Hatzichristou, Dimitrios

Hayden, F.G.

He, Meizi

Heath, Iona

Hébert, Paul Clarence

Hebert, Philip Charles

Hébert-Croteau, Nicole

Heckman, George A.

Hegele, Robert

Hegney, Desley Gail

Heidegger, Thomas

Henderson, Isabel

Henderson, James

Hendra, Tim

Henkel, Ralf

Hennen, Brian

Henschke, C.I.

Herlitz, Johan

Hernández, Adrián V.

Hewson, P.J .

Higgins, Rosemary D.

Higginson, Lyall A. J.

Hill, John S.

Hillemeier, Marianne M.

Hills, Marcia

Hilsden, Robert

Hingson, Ralph

Hirsh, Vera

Hnid, Karim

Hobbs, Simon

Hobson, RW

Hodge, Matthew J.

Hodsman, Anthony

Hoff, Geir

Hofmann, Adam

Holick, Michael

Holshouser, B.A.

Holt, Tim

Hookey, Lawrence

Hooper, Jon

Houston, Mark C.

Howes, Daniel William

Hsiao, S.H.

Hsu, Chiu-Hsieh

Huang, Allen

Hueston, W.J.

Hughes, John

Hughes, Merrilee

Huikuri, Heikki V.

Hull, Russell D.

Hunold, Peter

Hurst, Samantha

Hutten-Czapski, Peter

Hux, Janet Elizabeth

Huynh, Thao

Hwang, Stephen Wesley

Ikonomidis, Ignatios

Ioannidis, John P.A.

Iqbal, Nayyar

Isaac-Renton, Judith L.

Ishak, Jack

Izzo, Angelo A.

Jack, Susan

Jackson, Jeffrey L.

Jacobs, Elizabeth 
Klatt, Edward

Klebanoff, Mark

Klonsky, E.D.

Knake, S.

Koehn, Peter H.

Komsta, Elizabeth Maria

Kontorinis, Nick

Kontos, Michael C.

Koren, Gideon

Kozyrskyj, Anita L.

Krajden, Mel

Krakoff, Lawrence

Kraman, Steve S.

Kravcik, Steve

Kreiger, N.

Krix, Martin

Kulminski, A.

Kunik, Mark

Kurmis, Andrew P.

Kushel, M.

Kwok, Matthew M.K.

Kwon, Brian

Kwong, Jeff

Kyaw, Moe $\mathrm{H}$.

Labinaz, Marino

Lacroix, Thomas Bernard

Ladouceur, Robert

la Fleur, Philip

Lagacé-Wiens, P.R.

Lakatos, Peter Laszlo

Lalonde, André B.

Lalonde, Lyne

Lambert, Bernard

Lang, Eddy S.

Langholz, Ebbe

Langley, Joanne Marie

Lapinsky, Stephen E.

Laredo, Sheila

Larsson, Margareta

Lau, David C.W.

Laupacis, Andreas

Laurenson, M.C.

Lawlor, Peter G.

Lawson, S.R.

Le May, Michel

LeBlanc, John Charles

Leblanc, Richard

Lee, Douglas S.

Lee, Robert C.

Lee, W.L.

Leech, Judy

Leenaars, A.A.

Légaré, Jean-François

Leigh, J.P.

Leone, Marc

Leong, Wendy A.

Leonoff, Arthur

Lepnurm, Rein

Leung, Gabriel M.

Levett, Paul N.

Levine, David

Levy, Adrian

Levy, Robert Daniel

Levy, Tally

Lewis, Steven

Lexchin, Joel

Li, Yihong
Liberati, Alessandro

Liberman, Moishe

Liebeskind, David S.

Lim, Wendy

Lindsay, Robert M.

Lipshultz, Steven E.

Lipsitz, Joshua Dan

Liu, J.P.

Liu, Linda

Liu, S.

Livermore, David M.

Lobato, M.N.

Lofsky, Stanley

Lofters, Aisha

Logroscino, Giancarlo

Lohmann, Reinhard C.

Long, Richard

Longo, Christopher John

Lopatka, Harold

Lopez-Escamez, Jose A.

Loria, Paola

Lorimer, John Wilson

Louie, Marie

Louie, Thomas J.

Louw, Jacob A.

Lugon, Jocemir Ronaldo

Luijendijk, Hendrika

Luo, Zhong-Cheng

Lynge, Elsebeth

Lysenyuk, Victor

McAvay, Gail J.

McCall, Maureen Dolores

McClean, Karen

McCloskey, B.A.

McCord, James

McCullough, Peter A.

McCusker, Jane

Macdonald, Mary Ellen

MacDonald, Neil

McDonald, Sarah D.

McDowell, Ian

McEwen, Andy

McGrail, Kimberlyn Marie

McGregor, Maurice

McIntosh, Greg

McIntyre, Lynn

McKendry, Robert J.R.

McKenney, James

McKinney, Veronica

MacKnight, C.R.

McLaughlin, Joseph Kevin

MacLean, J. Dick

MacLeod, Stuart M.

McMillan, Douglas Donald

McNeil, Shelly

McNeill, Paul M.

McPherson, David D.

McPherson, Ruth

MacStravick, Scott

Magann, E.F.

Maggi, Stefania

Maggon, Krishan

Magura, Steve

Mahaffey, Peter J.

Maheux, Pierre

Mahle, William

Mailman, Tim
Mallin, Robert

Mallon, William T.

Mandel, J.

Manns, Braden

Manuel, D.G.G.

Marczewski, K.T.

Marek, Karen Dorman

Mark, Daniel B.

Markman, M.

Marks, Meridith

Marra, Carlo

Marras, Theodore K.

Marrie, Thomas J.

Marsden, Joanne

Marshall, Alison 1.

Marshall, Shawn Calder

Martensson, L.

Martin, Ian

Martin, Shelley

Martin, W.R.W.

Martinez, Alma Marinz

Marusic, Ana

Masip, J.

Mason, Robin

Mathews, Maria

Matlow, Anne G.

Matsui, Doreen Miyako

Mauri, Davide

Mawudeku, Abla

Mayers, Irvin

Mazer, Bruce

Mazur, Dennis J.

Mazzucco, Sara

Mecocci, Patrizia

Meissner, Helen I.

Meltzer, Sara J.

Ménard, Henri André

Mencke, Thomas

Mendelson, Michael

Menon, Devidas

Menzies, Dick

Merchant, Anwar Tajdin

Merchant, Roland Clayton

Miller, Anthony Bernard

Miller, Wendy M.

Mills, Edward J.

Minde, $\mathrm{K}$.

Mintzes, Barbara

Miro, O.

Mitchell, Ian

Mittleman, Murray A.

Moayyedi, Paul

Moffatt, M.E.K.

Mojtabai, Ramin

Moltzan, C.

Monreal, Manuel

Montgomery, Alan

Montgomery, Mark

Moon, James C.

Moore, C.L.

Moore, Katherine N.

Moore, R.A.

Moores, David G.

Morana, Giovanni

Moreau, Caroline

Morrison, Katherine Mary

Mosher, Dianne Patricia
Mosimann, Urs Peter

Mounier-Jack, Sandra

Moyer, Alwyn

Mückter, Harald

Munoz, David

Munoz, F.M

Muscat, J.E.

Naidoo, Poobalan

Nakano, Shinichi

Naleway, A.L.

Nash, Denis

Natarajan, Madhu

Nathan, Howard J.

Nava, Stefano

Neutel, C. Ineke

Newbold, K. Bruce

$\mathrm{Ng}$, Dominic

Ng, Nawi

$\mathrm{Ng}, \mathrm{T}$.

Nguyen, Mai

Nicolle, Lindsay

Nienaber, Christoph A.

Nikièma, Béatrice

Nilstun, Tore

Ni Mhurchu, Cliona

Nisker, Jeffrey Allan

Nissapatorn, V.

Nixon, Mary Kay

Nordentoft, Merete

Norton, Scott A.

Nuermberger, E.

Nyatanga, Brian

Oho, Takahiko

Oliver, Lisa $\mathrm{N}$.

Olsen, J.

Olshen, Elyse

Olson, Carin M.

Olson, Karin

Onder, Graziano

O'Neill, Blair

Ong, Ken

Oppel, Lloyd Bruce

O'Shaugnessy, D.F.

Otley, Anthony

Ott, Brian

Otto, Suzie J.

Oude Voshaar, R.C.

Padmore, Ruth

Padwal, Raj

Palareti, G.

Palferman, T.G.

Papineau, Elisabeth

Parmar, Malvinder Singh

Parry, Steve W.

Parshuram, Christopher S.

Partin, Melissa R.

Paskett, Electra

Paterson, B.

Paul, M.

Pauls, Merril Andrew

Pearce, Mark Allan

Pedersen, A.M.L.

Peele, P.B.

Pellicelli, Adriano M.

Peltekian, Kevork Minas

Pengo, Vittorio

Pépin, Jacques

Pereg, David

Peris, Ketty

Perreault, Sylvie

Perrella, Alessandro

Perron, Stéphane

Person, Bobbie

Petak, Steven

Petersen, M.L.

Peterson, Robert

Pettifor, John Morley

Phillips, C.O.

Phillips, Peter

Phypers, Melissa

Pickett, William

Pickles, $\mathrm{H}$.

Piedra, Pedro Antonio

Pijak, Michal

Pilote, Louise

Pimlott, Nicholas J.G.

Pinheiro, C.H.da J.

Pinkerton, S.D. 
Richards, Mark

Richter, Magdalena S.

Ridde, Valéry

Riedel, Stefan

Rieder, Michael J.

Rioux, France Marie

Ritmeijer, Koert

Rivest, Paul

Roberts, Harold R.

Roberts, James M.

Roberts, $\mathrm{S}$.

Robertson Blackmore, E.

Robinson, D.

Robinson, Elizabeth

Rocca, Corinne

Rockwood, Kenneth John

Rodd, Celia Jane

Rodrigo, Ramón

Rodway, George W.

Roland, M.E.

Roper, M.E.A.

Rosengart, M.R.

Rosin, A.

Rosser, Walter W.

Rothenberg, Marc E.

Rouleau, Jean-Lucien

Rowe, Brian $\mathrm{H}$.

Rowley, Kevin

Roy, Marc-André

Royall, Donald R.

Rudner, Robert

Rughani, Anand

Ruhe, J.J.

Russell, Barbara J.

Russell, Cory

Russell-Mayhew, Shelly

Ryan, A.S.

Ryu, Jay $\mathrm{H}$.

Sachs, Magda

Sackner-Bernstein, J.

Sahin, Mehmet

Sahpazova, Emilija

Salisbury, Chris

Salomaa, Veikko

Salpeter, Shelley

Salvini, Camilla

Samartzis, Dino

Samsa, G.P.

Sand, Peter Kevin

Sander, Josemir

Sanders, James

Sandor, George

Sankaran, Koravangattu

Sapp, John L.

Sargent, Richard

Sarwar, Shahbaz C.

Sarwer, D.B.

Savory, John

Scagliotti, Giorgio

Schaafsma, Joseph

Schanzer, Dena

Scharhag, J.

Schattner, Ami

Schaub, B.

Schaubel, Douglas Earl

Schers, $\mathrm{H}$.

Schindler, Christoph
Schleinitz, M.D.

Schmitz, Norbert

Schneider, Bryan James

Schramm, David

Schroth, Robert J. John

Schull, Michael John

Schultz, Jennifer

Schumann, A.

Schuppli, Catherine Anne

Schuster, Richard

Schwab, Abraham

Schwam, E.

Schwappach, David

Schwartz, Faina

Schwartzman, Kevin

Scott, Jeff

Scott, W.G.

Scully, Hugh E. Edwards

Sears, Margaret Elizabeth

Seeman, Mary Violette

Seeman, Neil L.

Seet, Raymond C.S.

Segev, Re'em

Seguin, Louise

Selgelid, Michael J.

Sen-Chowdhry, Srijita

Senecal, Pierre Etienne

Senf, J.H.

Sengupta, Sohini

Senthilselvan, A.

Servillo, G.

Shahin, Rita

Shanafelt, Tait

Shankaran, Seetha

Sharma, Arya M.

Sharma, Om P.

Sharma, Sat

Shave, R.E.

Sheldon, Claire Alexis

Shemie, Sam David

Shenoy, Chetan

Sherman, K.

Sherwin, Susan

Shibata, Marcelo C.

Shirom, A.

Shojania, A. Majid

Short, Andrew Liam

Shuchman, Miriam

Shulman, H. Mitchell

Siemens, David Robert

Silva, Matthew A.

Silva, Paulo Vasconcellos

Siminoski, Kerry

Simons, Leon

Simonsick, Eleanor Marie

Simpson, C.S.

Simpson, J.C.

Sims, Erika J.

Simunovic, Marko R.I.

Singh, Sarabjeet

Sinkin, Robert A.A.

Sitar, Daniel S.

Skea, Susan

Skinner, Chris

Skyler, J.S.

Small, Dan

Smith, Eldon R.
Smith, Robert

Smits, T.

Smylie, Janet K.

Snell, Linda S.

Sobolev, Boris G.

Sojka, Peter

Solymoss, Susan

Somali, M.

Soto Mas, F.G.

Spaulding, Anne

Spencer, Adam

Spiers, Judith Ann

Springer, Sandra Ann

Spurll, Gwen

Stanbrook, Matthew

Stanwick, Richard

Stephen

Stanworth, Simon

Starfield, Barbara

Stark, Damien

Steele, Margaret

Steen, V.

Steinberg, Daniel

Steinke, Douglas

Steinman, Mike

Stephens, Carolyn

Stewart, Lygia

Stobart, Kent

Stokes, Aidan

Stossel, Tom

Strang, Robert

Stukenborg, G.J.

Suh, Kathryn N.

Sutton, Robert

Swadron, Stuart

Swor, Robert A.

Szafran, Olga

Taddio, Anna

Taitz, J.

Takahashi, Paul

Tamblyn, Robyn M.

Tanenbaum, S.J.

Tang, Anthony

Tanko, Laszlo B.

Tannenbaum, Hyman

Targher, Giovanni

Targownik, Laura Ellyn

Tator, Charles H.

Taylor, James

Teitel, Jerome $M$.

Tempier, Raymond

Ten Cate, Olle Th J.

Tenenbein, Milton

Teo, Koon K.

Termorshuizen, F.

Thabane, Lehana

Thabane, Marroon

Thirthalli, Jagadisha

Thompson, E.C.

Thompson, Paul

Thomsen, Reimar Wernich

Thomson, Colin John

Thon, Jonathan Noah

Thongboonkerd, Visith

Thoresen, Marianne

Tinmouth, Jill

Tobe, Sheldon W.
Tokola, Kari

Tomkowski, W.Z.

Torri, Valter

Toth, Ellen Louise

Toth, Peter Paul

Tough, Suzanne C.

Towns, Dale

Trampuz, Andrej

Trudel, Guy

Tsao, Jennie

Tseng, Chin-Hsiao

Tsironi, Maria

Tu, Jack Ven

Tucker, Sheridan

Tugwell, Peter

Tulloh, Lou

Tuokko, H.

Turnbull, Jeffrey Michael

Turnbull, Robert G.

Tzeng, Huey Ming

Ulrich, Connie

Upshur, Ross E.G.

Ur, Ehud

Urbach, David Robert

Uzzan, Bernard

Vakil, Nimish

Valiquette, Luc

Valois, Maria

van den Heuvel, J.

van der Jagt, Richard

Van der Windt, Danielle

Van Jaarsveld, C.H.M.

Van Rosendaal, Guido

Van Tinteren, $\mathrm{H}$.

Van Walraven, Carl

Van Weel, C.

van Zyl, L.S.T.

Vas, Jorge

Vasterling, Jennifer

Vaudry, Wendy

Veitch, Craig

Velez-McEvoy, Margarita

Vento, Sandro

Ventres, William

Verhulst, Lorne Antony

Verma, Sarita

Verma, Subodh

Veugelers, Paul Joannes

Vickers, Andrew J.

Vickers, David

Victor, Gary

Vincer, Michael John

Virani, Sean A

Voeller, Heinz

Volker, Schöffl

Vorobioff, J.D.

Voruganti, Lakshmi Panth

Voyer, Philippe

Wada, Jun

Wagner, Todd $\mathrm{H}$.

Wahlander, Karin F.C.

Wainberg, Mark

Waldman, Deane

Walker, Karen Zell

Walsh, John Peter

Walsh, Stewart Redmond

Walsh, T.L.

Wang, F.-L.

Wang, Feng

Wang, Lu

Warburton, Darren E.R.

Ward, Brian James

Ward, Leanne

Ward, Stephen John

Wardman, A.E. Dennis

Wasserman, D.

Wazni, Oussama

Wear, Delese

Webb, John

Webby, Richard

Weber, Matthias M.

Weese, Scott

Weiner, W.J.

Weir, Matthew

Weisberg, E.

Weiss, Ram

Wells, Phil Stephen

Wen, Chi Pang 